\title{
Utilization of secondary-treated wastewater for the production of freshwater microalgae
}

C. Gómez-Serrano, M.M Morales-Amaral, F.G. Acién, R. Escudero, J.M. FernándezSevilla, E. Molina-Grima.

Department of Chemical Engineering, University of Almería, 04120 Almería, Spain.

Author for correspondence: F. Gabriel Acién Fernández

Department of Chemical Engineering, University of Almería

Carretera Sacramento s/n, E04120, Almería, Spain

Telephone: +34 950015443; Fax: +34 950015484; e-mail: facien@ual.es

KEYWORDS: microalgae, wastewater, nutrient limitation, productivity, biochemical composition, light efficiency 


\begin{abstract}
In this work we studied the potential use of secondary-treated wastewater as nutrient source in the production of freshwater microalgae strains. Experiments were performed indoors in semicontinuous mode, at $0.3 \mathrm{day}^{-1}$, simulating outdoor conditions. We demonstrated that all the tested strains can be produced by using only secondary-treated wastewater as the nutrient source. The utilization of secondary-treated wastewater imposes nutrient-limiting conditions, with maximal biomass productivity dropping to $0.5 \mathrm{~g} \cdot 1^{-1} \cdot$ day $^{-1}$ and modifies the biochemical composition of the biomass by increasing the amount of lipids and carbohydrates while reducing the biomass protein content. We measured fatty acids content and productivity of up to $25 \% \mathrm{~d}$.wt. and $110 \mathrm{mg} \cdot \mathrm{l}^{-1} \cdot \mathrm{day}^{-1}$, respectively. We demonstrated that all the tested strains were capable of completely removing the nitrogen and phosphorus contained in the secondary-treated wastewater, and while the use of this effluent reduced the cells' photosynthetic efficiency, the nitrogen and phosphorus coefficient yield increased. Muriellopsis sp. and S. subpicatus were selected as the most promising strains for outdoor production using secondarytreated wastewater as the culture medium; this was not only because of their high productivity but also their photosynthetic efficiency, of up to $2.5 \%$, along with nutrient coefficient yields of up to $96 \mathrm{~g}_{\text {biomass }} \cdot \mathrm{g}_{\mathrm{N}}{ }^{-1}$ and $166 \mathrm{~g}_{\text {biomass }} \cdot \mathrm{g}_{\mathrm{P}}{ }^{-1}$. Coupling microalgae production processes to tertiary treatment in wastewater treatment plants makes it possible to recover nutrients contained in the water and to produce valuable biomass, especially where nutrient removal is required prior to wastewater discharge.
\end{abstract}




\section{Introduction}

Microalgae have been proposed for a multitude of applications over the decades, but as yet only processes focused on high-value products for human use are commercially established. However, with global warming and the increase in oil prices, greater use of renewable energy is required. For this reason, much of the current microalgae research is focused on biofuel production because of these microorganisms' high potential energy yield per hectare (Chisti 2007; Mata et al. 2010). Microalgae biomass can be used to produce a wide range of biofuels such as bio-diesel, bio-ethanol, bio-methane and bio-hydrogen (Rosenberg et al. 2008; Schenk et al. 2008) although the process has not yet been performed on an industrial scale. One of the reasons for this is a lack of the great quantities of feedstock required to produce the considerable amounts of biomass demanded by these market, not to mention the necessary water and nutrients such as nitrogen and phosphorus (Acién et al. 2012). On the other hand, wastewater treatment is a large industry managing enormous volumes of water and nutrients, which include nitrogen and phosphorus. Consequently, the possibility exists of coupling wastewater treatment to microalgae production, an avenue that has already been explored (Hernández and Olguín 2002; Olguín 2003; Muñoz and Guieysse 2006; Pittman et al. 2011; Park et al. 2011; Li et al. 2011; Olguín 2012).

The wastewater industry is subject to ever more restrictive regulations, making it essential to develop more efficient processes capable of meeting the international regulations governing discharged water quality, while at the same time consuming less energy and enhancing the recovery of nutrients, such as nitrogen and phosphorus, from wastewater. It is important to note that European Directive 98/15/EC, which regulates water release, establishes a limit of $10 \mathrm{mg} \cdot 1^{-1}$ for nitrogen and $1 \mathrm{mg} \cdot \mathrm{l}^{-1}$ for phosphorus. Conventional activated sludge processes transform wastewater contaminants into nonvaluable products such as $\mathrm{N}_{2}$ and $\mathrm{CO}_{2}$, and in the process consume large amounts of energy $\left(0.5-1.0 \mathrm{kWh} \cdot \mathrm{m}^{-3}\right)$, whereas the utilization of microalgae-bacteria consortiums allows one to recover the nitrogen and phosphorus contained in the wastewater yet consume far less power $\left(0.1-0.2 \mathrm{kWh} \cdot \mathrm{m}^{-3}\right)$ (Aqualia, personal communication). Indeed, it has been reported that microalgal production costs using wastewater treatment can be covered by the wastewater treatment plant capital and operating costs themselves and thus cause significantly less environmental impact compared to cultivation systems using freshwater and fertilizer (Park et al. 2011). Moreover, life cycle analyses (LCA) 
concluded that the environmental burden associated with microalgae production for biofuels could be considerably reduced by coupling biomass cultivation and processing to wastewater treatment and industrial $\mathrm{CO}_{2}$ capture (Collet et al. 2013; Lardon et al. 2009).

However, the utilization of microalgae-bacteria consortiums requires large surface areas and favorable environmental conditions, meaning this technology cannot universally replace current processes based on activated sludge. Moreover, the replacement of present-day wastewater treatment processes would take considerable time as it is also necessary to develop strategies which enhance the capability of existing wastewater treatment plants so that they can comply with more restrictive regulations. In this regard, coupling a microalgae-based process as a tertiary treatment in existing wastewater treatment plants provides a reasonable alternative. The use of secondarytreated wastewater in microalgae production allows the nitrogen and phosphorus contained within it to be reused. In addition, the low nitrogen and phosphorus concentrations found in this effluent cause stress due to nutrient deficiency; this, in turn, enhances the synthesis and accumulation of large amounts of triglycerides useful in biodiesel production (Chisti 2007; Spolaore et al. 2006). Nonetheless, wastewater may also contain certain constituents that potentially inhibit microalgae growth such as urea, ammonium, organic acids, phenols and pesticides, which at high concentrations might limit the use of these effluents for microalgae production (Kumar et al. 2010). To examine this, a study needs to be undertaken which looks at individual wastewaters from each wastewater treatment plant in order to evaluate their subsequent use as culture mediums.

The most commonly used microalgae in wastewater treatment processes are the genera Chlorella and Scenedesmus, although Muriellopsis and Botryococcus strains have also been tested (Gómez-Serrano et al. 2013; Sutherland et al. 2014). There are no strains used specifically in wastewater treatment; indeed, any strain combining high biomass productivity and tolerance to outdoor conditions can be utilized. Nonetheless, a strain's tolerance to a particular wastewater used as the culture medium has to be verified. Moreover, identifying the most productive and efficient strains can enhance process yield and reliability. For this, in addition to productivity and biochemical composition, other cell properties such as cell weight, light utilization efficiency and nutrient requirements must be considered (Gómez-Serrano et al. 2013). 
In this paper, we studied the performance of five different freshwater strains in secondary-treated wastewater, as well as in Arnon medium, used as the standard. Experiments were performed indoors simulating outdoor conditions in order to maximize the applicability of results. Experiments were performed in semicontinuous mode as required for large-scale production. The aim was to determine the feasibility of using secondary-treated wastewater as the sole water and nutrient source in large-scale microalgae production and to identify those strains with the greatest potential. Moreover, we studied the phenomena which occurred while operating under these conditions as a prior step to developing the process on a large scale. The coupling of microalgae production processes to tertiary treatment in wastewater treatment plants allows one to recover the nutrients contained in the wastewater and to produce valuable biomass, especially where nutrient removal is required prior to wastewater discharge.

\section{Materials and methods}

\section{Microorganisms and culture media}

Five different freshwater microalgae strains were used: Chlorella vulgaris (SAG 21112), Chlorella fusca (SAG 211-8), Chlorella sp (SAG 1.8), Scenedesmus subpicatus (SAG 54.80) and Pseudokirchneriella subcapitata (SAG 61.81). The strains from the Chlorella genus are characterized by rapid growth and success in large-scale systems. They are frequently used in wastewater treatment and are also potentially useful in producing large amounts of lipids. S. subpicatus was also selected for its rapid growth rate and tolerance to adverse culture conditions. Pseudokirchneriella subcapitata was chosen for its use in environmental risk assessment where it serves as a control organism in toxicity studies, therefore making it useful for evaluating adverse effects under any particular culture condition. The standard culture medium used was Arnon medium (Arnon et al. 1974) enriched with $0.850 \mathrm{~g} \cdot 1^{-1}$ of $\mathrm{NaNO}_{3}$. Secondary-treated wastewater (STWW) was obtained from a real wastewater treatment plant located in Almería (Spain), the property of Aqualia. The STWW was collected after the last process decanter, once biological depuration by active sludge treatment had been performed, prior to release. The wastewater was filtered solely using $100 \mu \mathrm{m}$ pore size filters to remove solids, no additional sterilization or treatment was performed. 


\section{Photobioreactors and culture conditions}

Experiments were performed in 12 bubble-column-type reactors with spherical bases (3 $\mathrm{cm}$ in diameter and $45 \mathrm{~cm}$ in height with a $300 \mathrm{ml}$ capacity), filled with $250 \mathrm{ml}$ of culture. Each reactor was aerated at $0.2 \mathrm{v} / \mathrm{v} / \mathrm{min}$, with $\mathrm{CO}_{2}$ injected on demand $(\mathrm{pH}=8)$ into the air flow entering each reactor via a solenoid valve controlled by a $\mathrm{pH}$ sensor. The reactors were artificially illuminated using eight $28 \mathrm{~W}$ fluorescent tubes (Philips Daylight T5), positioned horizontally $1 \mathrm{~cm}$ apart from each other and $4 \mathrm{~cm}$ from the cultures. Illumination was performed using a solar light/dark cycle. The maximal irradiance inside the columns in the absence of cells was $1850 \mu \mathrm{E} \cdot \mathrm{m}^{-2} \cdot \mathrm{s}^{-1}$, measured using a SQS-100 Walz GmbH spherical quantum sensor (Effeltrich, Germany). The culture temperature was kept at $25^{\circ} \mathrm{C}$ by controlling the room temperature where the reactors were located; the maximum temperature increase due to light absorption was $1.5^{\circ} \mathrm{C}$ at the highest cell densities; and this was only during lighting hours.

Experiments were performed simultaneously in all reactors, which were inoculated with $10 \%$ of the culture volume from the same standard inoculum. Following this, the reactors were operated in batch mode for 6 days, after which they were operated in semicontinuous mode. For this, every day we harvested $30 \%$ of culture volume and replaced it with fresh culture medium. Semicontinuous culture was repeated daily until the culture volume was renewed at least three times, and the biomass concentration remained constant for at least three days. Harvested biomass and supernatant were used for analytical determinations. In each experiment, identical culture conditions were assayed in three reactors, thus each experimental condition was assayed in triplicate. The reactors were washed after each experiment and re-inoculated for the next experiment so as to avoid phenomena accumulation from previous conditions.

\section{Biomass concentration, light utilization and nutrient efficiency}

Cell density was measured with a Neubauer chamber using an Olympus CH20 microscope (Olympus Corp., USA). The dry weight biomass concentration ( $\mathrm{Cb}$ ) was measured by filtering $50 \mathrm{ml}$ of culture through $0.45 \mu \mathrm{m}$ filters and drying it in an oven at $80^{\circ} \mathrm{C}$ for $24 \mathrm{~h}$. Optimal quantum yield measurements were taken with a Junior-PAM fluorimeter (Walz, Germany). The biomass extinction coefficient (Ka) was calculated from the average absorption value in the visible range $(400-700 \mathrm{~nm})$, measured in a double beam Helios Alpha spectrophotometer. The extinction coefficient was calculated 
by dividing the average absorption by the biomass concentration $(\mathrm{Cb})$ and the cuvette light path (p) (Equation 1).

$\mathrm{Ka}=\frac{\overline{\mathrm{Abs}}_{400-700}}{\mathrm{C}_{\mathrm{b}} \cdot \mathrm{p}}$

Equation 1

The average irradiance at which cells are exposed inside a culture (Iav) is a function of irradiance in the absence of cells (Io), the biomass extinction coefficient (Ka), the biomass concentration $(\mathrm{Cb})$ and the light path inside the reactor (p). It can be approximated using Equation 2 (Molina-Grima et al. 1997).

$\operatorname{Iav}=\frac{\mathrm{I}_{\mathrm{o}}}{\mathrm{K}_{\mathrm{a}} \mathrm{C}_{\mathrm{b}} \cdot \mathrm{p}} \cdot\left(1-\exp \left(-\mathrm{K}_{\mathrm{a}} \mathrm{C}_{\mathrm{b}} \cdot \mathrm{p}\right)\right)$

Equation 2

Quantum yield $\left(\Psi_{\mathrm{E}}\right)$ is defined in microalgal cultures as the amount of biomass generated by the unit of radiation (usually a mole of photons) absorbed by the culture. Since it represents the ratio of biomass generated to absorbed photon flux, it can be calculated using Equation 3 (Molina-Grima et al. 1997) as a function of the volumetric biomass productivity $(\mathrm{Pb})$ and the photon flux absorbed in the volume unit (Fvol). The photon flux absorbed through the reactor volume can be obtained from the average irradiance (Iav) on a culture volume; this can be calculated using Equation 4 (MolinaGrima et al. 1997).

$\Psi_{\mathrm{E}}=\frac{\mathrm{P}_{\mathrm{b}}}{\mathrm{F}_{\mathrm{vol}}}$

Equation 3

$\mathrm{F}_{\mathrm{vol}}=\mathrm{Iav} \cdot \mathrm{K}_{\mathrm{a}} \cdot \mathrm{C}_{\mathrm{b}}$

Equation 4

Photosynthetic efficiency was calculated as the ratio of energy stored in the biomass produced to energy impinging on the reactor surface (Equation 5). The combustion heat of the biomass $(\mathrm{H})$ was calculated considering the specific caloric value of the lipids $(38.9 \mathrm{~kJ} / \mathrm{g})$, proteins $(24 \mathrm{~kJ} / \mathrm{g})$ and carbohydrates $(16.6 \mathrm{~kJ} / \mathrm{g})$, and by knowing the biochemical composition of the biomass. This equation uses the volume to surface ratio $(\mathrm{V} / \mathrm{S})$ of the reactor, and the PAR to global ratio of light, which was $2 \mathrm{E} \cdot \mathrm{MJ}^{-1}$.

$P E=\frac{\mathrm{P}_{\mathrm{b}} \cdot \mathrm{H} \cdot \mathrm{V}}{\mathrm{I} \cdot \mathrm{S}}$

Equation 5

The nitrogen $\left(\mathrm{Y}_{\mathrm{b} / \mathrm{N}}\right)$ and phosphorus $\left(\mathrm{Y}_{\mathrm{b} / \mathrm{P}}\right)$ coefficient yields were respectively determined as the amount of nitrogen and phosphorus removed from the culture medium to the ratio of biomass produced (Equation 6, Equation 7). The nitrogen removed was calculated as the difference between the total nitrogen (TKN) in the reactor's inlet and outlet streams at the imposed dilution rate (D). The phosphorus 
removed was calculated as the difference between total phosphorus (TP) in the reactor's inlet and outlet streams at the imposed dilution rate (D).

$\begin{array}{lc}\mathrm{Y}_{\mathrm{b} / \mathrm{N}}=\mathrm{D} \frac{[\mathrm{TKN}]_{\text {inlet }}-[\mathrm{TKN}]_{\text {outlet }}}{\mathrm{Pb}} & \text { Equation 6 } \\ \mathrm{Y}_{\mathrm{b} / \mathrm{P}}=\mathrm{D} \frac{[\mathrm{TP}]_{\text {inlet }}-[\mathrm{TP}]_{\text {outlet }}}{\mathrm{Pb}} & \text { Equation 7 }\end{array}$

Analytical Methods

The chemical composition of the water samples was determined using the standard methods approved by the Spanish Ministry of Agriculture (Ministerio de Agricultura 1982). We determined sodium, calcium, potassium, magnesium, iron, copper, manganese and zinc by atomic absorption or emission. Phosphorus was measured by visible spectrophotometry through the phospho-vanado-molybdate complex. We quantified carbonates and bicarbonates by a volumetric method with $0.01 \mathrm{~N}$ sulphuric acid in $\mathrm{pH} 8.3$ - the carbonates with $1 \%$ phenolphthalein and $\mathrm{pH} 4$ and the bicarbonates with $1 \%$ methyl orange. Chloride was measured by a volumetric method with silver nitrate between $0.01 \mathrm{~N}$ and $1 \mathrm{~N}$, potassium chromate was used as the indicator (The Mohr Valuation). Nitrates were quantified spectrophotometrically between 220 and $275 \mathrm{~nm}$. Ammonium was measured by the Nessler reactive method. Boron was determined following a reaction with azomethine measured spectrophotometrically. We measured sulphates using a baric sulphate precipitate.

To determine the biochemical composition of the biomass, we quantified the lipids, proteins, carbohydrates and ash content, in addition to the fatty acids content. Lipids were determined gravimetrically from an extract obtained with chloroform:methanol $(2: 1)(\mathrm{v} / \mathrm{v})$ (Kochert 1978). Fatty acids were determined by gas chromatography (Rodríguez-Ruiz, et al. 1998). The protein content was determined using the Lowry method. Total ash was determined by incineration of a $0.5 \mathrm{~g}$ sample in an oven at $450^{\circ} \mathrm{C}$ for $48 \mathrm{~h}$. Finally, we estimated carbohydrates as the difference (out of a hundred) after subtracting the lipid, protein and ash content.

\section{Results}

We studied the possibility of producing selected freshwater strains using only wastewater from secondary treatment (STWW) as the nutrient source. In addition, Muriellopsis sp. data from a previous study were also considered (Gómez-Serrano et al. 
2013). We carried out semicontinuous cultures at a dilution rate of 0.3 day $^{-1}$ with each of the selected strains, using Arnon medium and secondary treated wastewater from a real wastewater treatment plant (Almería, Spain) as the culture medium. Operating in semicontinuous mode, we obtained steady states from which the strains' yields were evaluated in terms of biomass production, cell characteristics, light utilization efficiency, biomass composition, nutrient removal capacity, etc. - all of which are necessary to adequately evaluate a strains' performance in the culture mediums used. The compositions of the culture mediums used are shown in table 1. Both mediums contained relevant concentrations of minor elements such as $\mathrm{Fe}, \mathrm{Mg}, \mathrm{Mn}$, etc. - all of them necessary for microalgae production. Regarding major compounds, the Arnon medium contained high concentrations of nitrogen and phosphorus, at 150 and 145 $\mathrm{mg} \cdot \mathrm{l}^{-1}$ respectively; with nitrogen in the form of nitrate. Conversely, in the secondarytreated wastewater, the nitrogen and phosphorus concentrations were much lower, at 17 and $10 \mathrm{mg} \cdot 1^{-1}$ respectively; with nitrogen mainly in the form of ammonium. Ammonium is more favorably assimilated by microalgae; however, at concentrations above 100 $\mathrm{mg} \cdot \mathrm{l}^{-1}$ it has been reported to be toxic (Collos and Harrison 2014). Regarding total inorganic carbon, both Arnon medium and STWW contained similar concentrations, of 48 and $43 \mathrm{mg} \cdot 1^{-1}$, respectively. According to these values, the C/N/P ratio of Arnon medium was 100/314/302 whereas for STWW, this ratio was equal to 100/40/23. Comparing these values with the expected ratio in the biomass, of $100 / 5 / 1$, it was concluded that both culture mediums were carbon limited, Arnon medium contained a significant excess of phosphorus with respect to the nitrogen content. STWW also contained an excess of phosphorus with respect to the nitrogen content, although lower than that observed in Arnon medium. The N/P ratio for Arnon and STWW was 1 and 1.7, respectively; the ratio cited in the literature varied between 3 and 30 (Park et al. 2009). In any case, table 1 demonstrates that Arnon medium provided excess nutrients whereas STWW contained low nutrient concentrations, thus we expect the cultures grown in this culture medium to be nutrient limited.

In spite of potential nutrient limitations imposed when using STWW, results show that all the strains could be produced in semicontinuous mode using only STWW as the nutrient source (Figure 1). These results confirm that STWW does not contain anything toxic to microalgae growth and that the minor component concentrations are sufficient to support the growth of the strains tested. The biomass concentrations achieved in steady state were higher when using Arnon medium than with STWW, therefore 
confirming nutrient limitation when STWW was used. The biomass concentration achieved using whichever culture mediums also varied according to the strain used, indicating that each had a different growth efficiency, even under the same nutrientlimited conditions. Using Arnon medium, the biomass concentration was higher than $1.5 \mathrm{~g} \cdot \mathrm{l}^{-1}$ for all strains, but using STWW, only cultures performed using Muriellopsis sp and $P$. subpicatus were higher than this value (Figure 1A). This is important not only because biomass concentration influences biomass productivity but also because high biomass concentrations improve culture stability, which is necessary for large-scale production. In terms of biomass productivity, Muriellopsis sp, C. fusca and $S$. subpicatus were the most productive strains when using Arnon medium, with productivities from 0.8 to $0.9 \mathrm{~g} \cdot \mathrm{l}^{-1} \cdot \mathrm{day}^{-1}$. However, when using STWW, the most productive strains were Muriellopsis sp and S. subpicatus, with biomass productivity reducing to $0.5 \mathrm{~g} \cdot 1^{-1} \cdot \mathrm{day}^{-1}$ (Figure 1B). C. vulgaris was the least productive strain in whichever culture medium used. The adverse culture conditions existing in the STWW culture medium were confirmed by analyzing the fluorescence of chlorophylls data (Figure $1 \mathrm{C}$ ). The $\mathrm{Fv} / \mathrm{Fm}$ values remained constant, and higher than 0.65 , for all strains when using Arnon medium; but using STWW, the Fv/Fm values dropped below this value in all the strains, indicating adverse culture conditions. Again, C. vulgaris showed the largest Fv/Fm value reduction, confirming this strain's elevated sensibility to STWW as the culture medium.

Regarding cell characteristics, we determined both the cell density and cell weight of the different strains when using Arnon and STWW as the culture mediums (Figure 2). Results showed that, whatever the strain, the cell density was higher using Arnon medium than STWW, in accordance with previous data on biomass concentrations (as dry weight) (Figure 2A). In contrast, the cell weight of all the strains was higher when using STWW than when using Arnon medium (Figure 2B). Cell density was maximal using Arnon medium for C. vulgaris, S. subpicatus and P. subcapitata, with values ranging from $1.20 \cdot 10^{8}$ to $1.29 \cdot 10^{8} \mathrm{cel} \cdot \mathrm{ml}^{-1}$; whereas using STWW, we measured a maximal cell density of $0.53 \cdot 10^{8} \mathrm{cel} \cdot \mathrm{ml}^{-1}$ for $S$. subpicatus. With regards to the cell weight using Arnon medium, Muriellopsis sp. and C. fusca showed the highest values, of $50 \mathrm{pg} \cdot \mathrm{cel}^{-1}$; whereas using STWW, Muriellopsis sp. again showed the highest value, of $75 \mathrm{pg} \cdot \mathrm{cel}^{-1}$. These variations suggest that the biochemical composition of the biomass modifies when produced in nutrient-sufficient conditions (Arnon medium) or nutrientlimited conditions (STWW). In any case, Muriellopsis sp. provided the largest cells, 
which is relevant for harvesting. The smallest cells were from $S$. subpicatus and $P$. subcapitata using both Arnon medium and STWW.

In addition to cell density and cell weight, we determined the extinction coefficient and average irradiance inside the cultures (Figure 3). The extinction coefficient quantifies the attenuation of light caused by the cells, whereas the average irradiance is the light availability required by the cells to maintain the dilution rate imposed. Results showed that Muriellopsis sp., S. subpicatus and P. subcapitata reduced their extinction coefficients when using STWW compared to those obtained using Arnon medium; whereas in the other strains tested, the extinction coefficient increased when using STWW as the culture medium (Figure 3A). An increased extinction coefficient implies greater light attenuation, which is potentially negative behavior. Theory suggests that biomass with a lower extinction coefficient is more appropriate for large-scale production because greater light availability inside the cultures can be achieved with the same external irradiance. Results showed that when using Arnon medium, the average irradiance inside the cultures was similar for all the strains tested, ranging from 31 to 54 $\mu \mathrm{E} \cdot \mathrm{m}^{-2} \cdot \mathrm{s}^{-1}$ (Figure 3B). Using STWW, the average irradiance was higher for all the strains although it increased especially for Muriellopsis sp., C. vulgaris and $P$. subcapitata. The results indicate that these strains need more light to maintain the imposed dilution rate when using STWW, thus reducing their efficiency more than for other strains. To evaluate this phenomenon, we measured the cells' capacity to utilize light off-line by determining the fluorescence of chlorophylls to light response curves (Figure 4). Results showed that, in Arnon medium, the Muriellopsis sp. strain exhibited the best performance, with higher electron transfer rate (ETR) values than other strains when measured with increasing light availability (Figure 4A). Conversely, C. vulgaris exhibited the worst efficiency in Arnon medium. When using STWW, the S. subpicatus and C. fusca strains performed best (Figure 4B). Muriellopsis sp. demonstrated intermediate efficiency whereas the $P$. subcapitata exhibited the lowest ETR values in STWW. Comparing the results from both culture mediums, we observed that the electron transfer rate (and so the performance of the photosynthetic apparatus) reduced in all the tested strains when STWW was used compared to values obtained using Arnon medium. Results also showed the cells were adapted to high-light conditions given the high irradiance used during the experiments - no photoinhibition at all was observed, even at irradiances above $1400 \mu \mathrm{E} \cdot \mathrm{m}^{-2} \cdot \mathrm{s}^{-1}$. 
Regarding the biochemical composition of the biomass, we measured the effect of using STWW as the culture medium on the total lipid, protein, carbohydrate and ash content of the lyophilized biomass obtained in steady state (Figure 5). Results showed that for all strains, the lipid and carbohydrate content of the biomass increased when using secondary-treated wastewater compared to values obtained using Arnon medium. The highest lipid content, of 35\%d.wt., was measured for P. subcapitata in STWW whereas the lipid content reduced to 24\%d.wt. for the same strain in Arnon medium (Figure 5A). In contrast, this same strain provided the lowest carbohydrate content, with maximal values of 46 and 32\%d.wt., when produced in STWW and Arnon medium, respectively (Figure 5C). Chlorella strains produced the largest carbohydrate content - up to 64\%d.wt. for Chlorella sp. using STWW. Unlike lipids and carbohydrates, the protein and ash content of the biomass reduced when using STWW rather than Arnon medium. P. subcapitata showed the highest protein content when produced in Arnon medium, of 34\%d.wt., but reduced to 13\%d.wt. in STWW (Figure 5B). The ash content was lower than $10 \%$ in all the strains and for all the culture conditions tested - it is relevant that the mean value reduced from 9\%d.wt when using Arnon medium to 5\%d.wt. when using STWW (Figure 5D). These results agree with the expected behavior of nitrogen-limited cultures (when using STWW) compared to nutrient-replete conditions (using Arnon medium). Therefore, under nitrogen-limited conditions, protein synthesis is limited and the energy from photosynthesis is stored as lipids or carbohydrates. Moreover, the lower ash content of STWW (compared to Arnon medium) results in a corresponding ash content reduction for biomass produced in STWW rather than Arnon medium.

In addition to the lipid content, we also measured the fatty acid content and productivity of the different strains, using both Arnon medium and STWW (Figure 6). We did this to determine the feasibility of using the resultant biomass to produce biodiesel by direct transesterification. Results were comparable to those observed for the lipid content - the fatty acid content of all the strains increased when using STWW rather than Arnon medium (Figure 6A). P. subcapitata and Muriellopsis sp. contained the largest proportion of fatty acids in the biomass, in both Arnon medium and STWW - the fatty acid content increased from 16 to $25 \%$ d.wt. in P. subcapitata, and from 12 to $22 \%$ d.wt. in Muriellopsis sp. when using Arnon medium and STWW, respectively (Figure 6A). All the Chlorella strains showed a similar fatty acid content, increasing from 10 to $15 \%$ in Arnon and STWW, respectively. In terms of productivity, both Muriellopsis sp and P. subcapitata had the highest fatty acid productivity when using Arnon medium; 
however, when using STWW, the most productive strains were Muriellopsis sp., $S$. subpicatus and P. subcapitata (Figure 6B). It is important to note that, even though $S$. subpicatus and P. subcapitata had the same fatty acid productivity using STWW, the highest fatty acid content was in $P$. subcapitata, making this strain more recommendable for biodiesel production.

Finally, to study the level of nutrient removal from the culture medium carried out by each strain, we determined the chemical composition at the reactors' water inlets and outlets for experiments performed using both Arnon medium and STWW. The main compound concentrations (total inorganic carbon, nitrogen and phosphorus) at the inlet and outlet of the reactors are shown in Figure 7. Results demonstrated that when using Arnon medium, the nitrogen at the inlet was not totally taken up by any of the strains, with nitrogen concentrations varying from $150 \mathrm{mg} \cdot 1^{-1}$ at the inlet to $8 \mathrm{mg} \cdot 1^{-1}$ at the outlet (Figure 7A). As nitrogen comes in the form of nitrate in Arnon medium, the uptake of such a large amount of nitrate releases a considerable concentration of hydroxyl ions into the culture medium, so the system requires more $\mathrm{CO}_{2}$ to control the $\mathrm{pH}$ (data not shown). This phenomenon explains the large increase of total inorganic carbon at the outlet compared to that at the inlet for whichever strain is used, increasing from 48 to $148 \mathrm{mg} \cdot 1^{-1}$ (Figure 7A). With regards to phosphorus, the concentration at the outlet was lower than at the inlet for all strain; nonetheless, in Arnon medium, due to the great excess of phosphorus, the concentration at the outlet was higher than $65 \mathrm{mg} \cdot \mathrm{l}^{-1}$ for all the strains (Figure 7A). European Directive 98/15/EC establishes a water release limit of $10 \mathrm{mg} \cdot 1^{-1}$ for nitrogen and $1 \mathrm{mg} \cdot 1^{-1}$ for phosphorus - thus the high outlet concentration of nitrogen and phosphorus means that the outlet medium cannot be released into the environment without treatment. When using STWW as the culture medium, we observed different behavior. In this case, the total nitrogen and phosphorus inlet concentration was low, at 17 and $10 \mathrm{mg} \cdot l^{-1}$ respectively; with outlet concentrations for both compounds being zero in all strain (Figure 7B). In addition, because nitrogen in STWW comes in the form of ammonium, its uptake does not modify the water's alkalinity, and so the total inorganic carbon at the reactor outlet is the same as at the reactor inlet (Figure 7B). Moreover, the system demanded less $\mathrm{CO}_{2}$ to control $\mathrm{pH}$ (data not shown). Consequently, it was demonstrated that the water leaving the reactors contained negligible concentrations of nitrogen and phosphorus, meaning it could be released safely into the environment when removing the microalgae biomass. 


\section{Discussion}

To produce large amounts of microalgae, large volumes of water and nutrients are required. Nonetheless, microalgae can be grown not only in freshwater but also in contaminated effluents, permitting the nitrogen and phosphorus contained within them to be used (Aslan and Kapdan 2006; Singh and Gu 2010). At the same time, wastewater treatment plants manage large volumes of water containing nitrogen and phosphorus. These plants are under mandatory obligation to develop more efficient processes which comply with discharged water quality requirements imposed by European Regulations (Directive 91/271/CE); as well as being obliged to use less energy and enhance nutrient recovery from the wastewater. Thus, the benefits of coupling wastewater treatment and microalgae production are evident, as have been previously reported (Muñoz and Guieysse 2006; Olguín 2003; Godos et al. 2010). Most of the previous works focus on the treatment of fouled wastewaters (from urban, industrial or animal-use sources) using microalgae-bacteria consortiums. Secondary-treated wastewater, on the other hand, is a clean effluent coming from wastewater treatment plants, which requires no tertiary treatment. It has a low chemical oxygen demand (COD) and no large bacterial contamination, yet still contains nitrogen and phosphorus concentrations limiting its direct release into the environment. Coupling microalgae production processes to tertiary treatment in these types of wastewater treatment plants allows the recovery of the nutrients contained in the water and, at the same time, the production of valuable biomass, especially where nutrient removal is required prior to wastewater discharge. The utilization of secondary-treated wastewater for microalgae production has the advantage of providing both water and nutrient availability in large amounts, as well as avoiding any inconvenience caused by organic matter in the untreated wastewater. Microalgae can bioremediate these effluents by removing the remaining nitrogen and phosphorus, thus avoiding eutrophication problems, as well as oxidizing any remaining compounds as a result of the oxygen oversaturation that takes place in the microalgae cultures.

The results reported here show that all the strains tested were capable of growing in secondary-treated wastewater (STWW) without additional nutrients although the productivity was lower compared to using standard culture medium caused by nutrient limitation in the STWW. The STWW's nitrogen and phosphorus content was 17 and 10 $\mathrm{mg} \cdot \mathrm{l}^{-1}$, respectively, much lower than the nitrogen and phosphorus content in Arnon 
medium (150 and $\left.145 \mathrm{mg} \cdot \mathrm{l}^{-1}\right)$ but above permitted limits for wastewater release into the environment - 10 and $1 \mathrm{mg} \cdot \mathrm{l}^{-1}$ for nitrogen and phosphorus, respectively. In spite of the low nutrient content of STWW, stable steady states were obtained in all the tested strains, even for biomass concentrations higher above $1.0 \mathrm{~g} \cdot \mathrm{l}^{-1}$ (Figure 1). The biomass productivities obtained using STWW were higher than $0.5 \mathrm{~g} \cdot \mathrm{l}^{-1} \cdot \mathrm{day}^{-1}$ when using Muriellopsis sp. and S. subpicatus, indicating that these strains are the most productive. Moreover, although the cells were stressed when using STWW as the culture medium, Fv/Fm values reduced compared to those measured using Arnon medium - no culture instability problems were observed and all performed adequately. The nutrient-limited conditions imposed by utilizing STWW as the culture medium also influenced cell weight and culture performance. The data reported here demonstrates that, under nutrient-limited conditions, the cell weight of all the tested strains increased (Figure 2); and the average irradiance that the cells were exposed to inside the cultures likewise increased (Figure 3). This is important because high irradiance has also been reported as a factor modifying the lipid content of some strains (Olguín et al. 2003; Rodolfi et al. 2009). Variation in cell weight is related to a modification in cell metabolism under nutrient-limited conditions. Hence, figure 5 demonstrates that the lipid and carbohydrate content increased in all the strains tested, whereas the protein and ash content decreased when operating under nutrient-limited conditions. The variation in average irradiance is related to the cells' ability to utilize light. Data in figure 4 show that when using STWW, the cells' capacity to perform photosynthesis diminished in all the strains, with reduced electron transfer rate values compared to those using Arnon medium. In spite of the adverse conditions imposed when using STWW as the culture medium, the results demonstrated that the biomass fatty acid content increased notably with no significant reduction in fatty acid productivity; indeed, it even increased in some strains such as Muriellopsis sp. and S. subpicatus (Figure 6). Finally, when using STWW as the culture medium, the data for nitrogen concentration at the reactors outlet showed that nitrogen and phosphorus were completely removed - meaning the water could be released safely into the environment; they also confirmed that the cultures became nutrient-limited (Figure 7).

The lower biomass productivity obtained when using STWW versus Arnon medium can be increased by supplying additional nutrients. Instead of using fertilizers, these nutrients can be obtained from sewage sludge, anaerobic effluents, animal waste or meat-processing wastewater (Olguín 2012; Olguín and Sánchez-Galván 2010). It was 
previously reported that Muriellopsis sp. increased its productivity from 0.5 to $0.9 \mathrm{~g} \cdot \mathrm{l}^{-1}$ when STWW was supplied with 33\% of nutrients contained in Arnon medium (GómezSerrano et al. 2013). Similarly, post-chlorinated wastewater has to be enriched with 5 $\mathrm{mM} \mathrm{NaNO}\left(70 \mathrm{mgN}_{\mathrm{N}} \cdot \mathrm{l}^{-1}\right)$ to maximize Chlorella $\mathrm{sp}$. productivity; whereas to improve the lipid content, it has to be enriched with up to $25 \mathrm{mM} \mathrm{NaNO}\left(350 \mathrm{mg}_{\mathrm{N}} \cdot \mathrm{l}^{-1}\right)$ (Mutanda et al. 2011). Botryococcus braunni was also successfully grown in secondarytreated sewage from domestic wastewaters, removing the nitrogen and phosphorus as well as accumulating higher lipid levels (17.85\%d.wt.) than those grown in a synthetic medium (Órpez et al. 2009). Nutrient limitation, and especially nitrogen limitation, has been reported as the most efficient approach to enhance microalgae lipid content (Huesemann and Benemann 2009). However, although the nitrogen-limited conditions can enhance lipid accumulation, it usually also leads to lower biomass productivity which, in the end, reduces overall lipid productivity. Data reported here demonstrate that the fatty acid productivity of Muriellopsis sp and S. subpicatus increased to 110 and $84 \mathrm{mg} \cdot 1^{-1} \cdot$ day $^{-1}$, respectively, under nutrient-limited conditions due to a greater increase in fatty acid content versus the reduction in productivity under these conditions. Conversely, the fatty acid productivity of C. vulgaris, C. fusca and P. subcapitata reduced to 30,53 and $77 \mathrm{mg} \cdot 1^{-1} \cdot \mathrm{day}^{-1}$, respectively, under nutrient-limited conditions, in spite of higher fatty acid contents due to lower biomass productivity under these conditions. Increases in lipid content of $25 \%$ have been reported for Nannochloris sp. at $14 \mathrm{mg}_{\mathrm{N}-\mathrm{NO} 3} \cdot \mathrm{l}^{-1}$ compared to those obtained at $140 \mathrm{mg}_{\mathrm{N}-\mathrm{NO} 3} \cdot \mathrm{l}^{-1}$; however, there was a tentimes reduction in biomass productivity (Takagi et al. 2000). With Botryococcus braunii, the lipid content increased from 10.96 to $17.85 \%$ d.wt. when using wastewater containing $1 \mathrm{mgN}_{\mathrm{N}} \cdot \mathrm{l}^{-1}$ instead of pure culture medium (Órpez et al. 2009). In Nannochloropsis sp., lipid productivity increased from 117 to $204 \mathrm{mg} \cdot \mathrm{l}^{-1} \cdot \mathrm{day}^{-1}$ under nitrogen-starved conditions (Rodolfi et al. 2009). The response of each strain to nutrient-limited conditions can differ; thus, within the same genus (e.g. Chlorella), some strains were found to accumulate starch under nitrogen-starved conditions whereas others accumulated neutral lipids ( $\mathrm{Hu} 2004)$.

Regarding the capacity for nutrient removal, results reported here confirm that all the tested strains were capable of completely removing the nitrogen and phosphorus contained in the STWW under the culture conditions imposed. Similar high absorption efficiencies have also been reported with other freshwater microalgae (Sydney et al. 2011; Craggs et al. 1997). With settled domestic sewage and secondary-treated 
domestic effluent, supplemented with settled swine wastewater, the nitrogen removal efficiency was in the $92-95 \%$ range, although the phosphate removal efficiency was lower, approximately $62-80 \%$ (Wang et al. 2010). Chlorella sorokiniana presented similar growth to the artificial medium in 4- to 8-times-diluted pig slurry, and severe inhibition was recorded on the biodegradation process in undiluted and twice-diluted wastewater (González et al. 2008). Using centrate as the culture medium, nutrient removal efficiencies of 11 and $45 \%$ for nitrogen and phosphorus, respectively, have been reported with Chlorella (Min et al. 2012), whereas using N. oleoabundans, ammonium removal was 90-95\% (Levine et al. 2011; Yang et al. 2011). Using primarysettled sewage, up to $90 \%$ of the nitrogen content and $80 \%$ of the phosphorus content was removed with C. vulgaris (Lau et al. 2014). In comparison, using urban wastewater, the removal of phosphorus was $80 \%$ for C. vulgaris and $83 \%$ for S. obliquus (RuizMarin et al. 2010). It is important to note that all the tested strains were capable of taking up ammonium as the nitrogen source without any adverse toxic effects, at least at the tested concentrations, which are much lower than the $100 \mathrm{mg} \cdot 1^{-1}$ reported to be toxic for microalgae strains (Collos and Harrison 2014).

To quantify the performance of different strains when using STWW and Arnon medium, in addition to biomass productivity and biochemical composition, two further parameters have to be included - the quantum yield, or light-use efficiency, and the nutrient-coefficient yield, or nutrient-use efficiency. These parameters allow us to quantify the efficiency of different strains in using the available resources, thus making it possible to estimate the most efficient strain to use outdoors at large scale under not such optimal culture conditions. In terms of light-use efficiency, considering the specific caloric value of lipids $(38.9 \mathrm{~kJ} / \mathrm{g})$, proteins $(24 \mathrm{~kJ} / \mathrm{g})$ and carbohydrates $(16.6$ $\mathrm{kJ} / \mathrm{g}$ ), and knowing the biochemical composition of the biomass, it is possible to determine photosynthetic efficiency as the percentage of energy stored in the biomass. Results show that under nutrient-sufficient conditions, Muriellopsis sp. and C. fusca were the most efficient, with quantum yield values up to $0.65 \mathrm{~g} \cdot \mathrm{E}^{-1}$ and photosynthetic efficiency of up to $2.5 \%$ being measured (Figure 8 ). However, under nutrient-limited conditions, using STWW as the culture medium, the light-utilization efficiency of all the strains reduced, with Muriellopsis sp. and S. subpicatus being the most efficient - we measured quantum yields up to $0.38 \mathrm{~g} \cdot \mathrm{E}^{-1}$ and photosynthetic efficiency of $1.5 \%$. It was previously reported that the quantum yield of Muriellopsis sp. reduced from $0.65 \mathrm{~g} \cdot \mathrm{E}^{-1}$ under no (or low) nitrogen limitation to $0.38 \mathrm{~g} \cdot \mathrm{E}^{-1}$ under severe nitrogen limitation, 
meaning this strain is less energy efficient under these conditions (Gómez-Serrano et al. 2013). Quantum yield has been reported as reaching maximum values of $0.65 \mathrm{~g} \cdot \mathrm{E}^{-1}$ when culturing Isochrysis galbana at low light under optimal conditions (Molina-Grima et al. 1997), reducing to $0.1 \mathrm{~g} \cdot \mathrm{E}^{-1}$ under high-light conditions, to the point of causing photoinhibition. Under nitrogen-sufficient conditions, the photosynthetic efficiency of Muriellopsis sp. was reported to reduce from $2.5 \%$ to $1.6 \%$ under severe nitrogen limitation (Gómez-Serrano et al. 2013). In addition, the maximum efficiency determined indoors can be assumed as the maximum value achievable outdoors; therefore this parameter is relevant for scaling-up purposes and to select the most robust strain for outdoor production.

Regarding the nutrient-coefficient yield, or nutrient-use efficiency, for nitrogen and phosphorus under the experimental conditions tested, this was calculated as the amount of biomass produced per mass unit of nitrogen and phosphorus taken up (Figure 9). Results show that under nutrient-limited conditions, using STWW as the culture medium, the nitrogen and phosphorus coefficient yields significantly increased with respect to those measured under nutrient-sufficient conditions, as when using Arnon medium. When considering a nitrogen and phosphorus content in the biomass of 5 and $1 \%$ d.wt., respectively, the nitrogen and phosphorus coefficient yield has to be 20 $\mathrm{g}_{\text {biomass }} \cdot \mathrm{g}_{\mathrm{N}}^{-1}$ and $100 \mathrm{~g}_{\text {biomass }} \cdot \mathrm{g}_{\mathrm{P}}{ }^{-1}$, respectively. Experimental values are close to these only under nutrient-sufficient conditions for all the strains tested. Under nutrient-limited conditions, Muriellopsis sp. and S. subpicatus showed the highest nitrogen and phosphorus coefficient yield values, up to $96 \mathrm{~g}_{\text {biomass }} \cdot \mathrm{g}_{\mathrm{N}}{ }^{-1}$ and $166 \mathrm{~g}_{\text {biomass }} \cdot \mathrm{g}_{\mathrm{P}}{ }^{-1}$. The data indicate that these strains are capable of producing larger amounts of biomass even under nutrient-limited conditions, making these strains the most efficient in terms of nutrient utilization.

Hence, the data reported here demonstrate that all the tested strains can be produced using only STWW as the culture medium but the efficiency of each varied. To select the most adequate strain for large-scale production, different criteria can be used. The most adequate criterion is economic profit; however, this requires the complete process to be defined and the results obtained to be validated at laboratory scale under outdoor conditions. Alternatively, we can use other criteria such as biomass productivity, fatty acid productivity, etc. but none of these take into account the efficiency of the strain (in terms of light or nutrient utilization) nor the ease of harvest. In order to compare the performance of different strains, the value of experimentally-obtained response 
variables was normalized, dividing it by the maximal value measured, thus obtaining a relative response variable comparison for each strain. Figure 10 shows the normalized values of the biomass' most relevant properties such as cell weight, biomass productivity, quantum yield, nitrogen and phosphorus coefficient yield and fatty acid content; in addition to the total value obtained by normalizing the sum of individual variables for each strain. According to this figure, we can conclude that under nutrientsufficient conditions (using Arnon medium), the most promising strains for outdoor production are Muriellopsis sp. and C. fusca, whereas under nutrient-limited conditions (using STWW), the most promising strains are Muriellopsis and S. subpicatus. We can obtain different results if pondered weights are applied to each factor; nonetheless, the selected strains demonstrated themselves as potentially useful for large-scale production using depurating STWW from real wastewater treatment plants.

Concluding, it was demonstrated that different freshwater strains can be produced using only secondary-treated wastewater (STWW) as the water and nutrient source. As a result of the low nitrogen and phosphorus concentration found in STWW, of 17 and 10 $\mathrm{mg} \cdot 1^{-1}$ respectively, the cultures become nutrient-limited thus reducing productivity to $0.5 \mathrm{~g} \cdot \mathrm{l}^{-1} \cdot \mathrm{day}^{-1}$ compared to that obtained under nutrient-sufficient conditions, of $0.9 \mathrm{~g} \cdot \mathrm{l}^{-}$

${ }^{1} \cdot$ day $^{-1}$. In spite of this reduction, the cultures were stable and the cells performed photosynthesis adequately, with the nitrogen and phosphorus being completely removed from the culture medium. Nutrient limitation enhances the lipid and carbohydrate content of the biomass up to 35 and $65 \%$ d.wt., respectively, depending on the strain, while the protein content reduces down to $15 \%$ d.wt. Although nutrient limitation reduces the photosynthetic performance of the cells because more light is needed to produce biomass, the nutrient coefficient yield increases under nutrient-limited conditions, thus the strains become more efficient at producing biomass with fewer nutrients availability. Considering the overall behavior of the strains tested, we selected both Muriellopsis sp. and S. subpicatus as the most promising for large-scale production using STWW as the culture medium.

\section{Acknowledgements}

This research was financed National Institute for Agriculture and Food Technology in Spain (INIA) by PURALGA project. We are most grateful to Aqualia S.A. for providing water samples and Estación Experimental Las Palmerillas of Fundación 
Cajamar for collaborating in this research. This research was supported by the Junta de Andalucía and the Plan Andaluz de Investigación (BIO 173).

\section{Ethical Statement /Conflict of Interest}

Authors mutually agree to submit this manuscript to Applied Microbiology and Biotechnology for publication. It is an original work that has not been previously submitted to Applied Microbiology and Biotechnology for publication. Research was supported by National Institute for Agriculture and Food Technology in Spain no conflict with whatever national or international organism existing. On this research no animals or humans are involved, and microorganisms used are not genetically modified.

\section{References}

Acién FG, Fernández JM, Magán JJ, Molina E (2012) Production cost of a real microalgae production plant and strategies to reduce it. Biotechnol Adv. 30 (6): $1344-1353$

Aslan S, Kapdan IK (2006) Batch kinetics of nitrogen and phosphorus removal from synthetic wastewater by algae. Ecol Eng 28: 64-70

Chisti Y (2007) Biodiesel from microalgae. Biotechnology Advances 25: 294-306

Collet P, Spinelli D, Lardon L, Hélias A, Steyer J-, Bernard O (2013) Life-Cycle Assessment of Microalgal-Based Biofuels. Biofuels from Algae : 287-312

Collos Y, Harrison PJ (2014) Acclimation and toxicity of high ammonium concentrations to unicellular algae. Mar Pollut Bull 80: 8-23

Craggs RJ, McAuley PJ, Smith VJ (1997) Wastewater nutrient removal by marine microalgae grown on a corrugated raceway. Water ResS 31: 1701-1707

Godos Id, Vargas VA, Blanco S, González MCG, Soto R, García-Encina PA, Becares E, Muñoz R (2010) A comparative evaluation of microalgae for the degradation of piggery wastewater under photosynthetic oxygenation. Bioresour Technol 101: $5150-5158$

Gómez-Serrano C, Escudero R, Morales MM, Figueroa FL, Fernández-Sevilla JM, Acién FG (2013) Use of secondary-treated wastewater for the production of Muriellopsis sp. Appl Microbiol Biotechnol 97: 2239-2249 
González C, Marciniak J, Villaverde S, García-Encina PA, Muñoz R (2008) Microalgae-based processes for the biodegradation of pretreated piggery wastewaters. Appl Microbiol Biotechnol 80: 891-898

Molina-Grima E, Camacho FG, Pérez JAS, Fernández FGA, Sevilla JMF (1997) Evaluation of photosynthetic efficiency in microalgal cultures using averaged irradiance. Enzyme and Microbial Technology 21: 375-381

Hernández E, Olguín EJ (2002) Biosorption of heavy metals influenced by the chemical composition of Spirulina sp. (Arthrospira) biomass. Environ Technol 23: 13691377

Hu Q (2004) Environmental effects on cell composition. In: Richmond A (ed) Handbook of microalgal culture: biotechnology and applied phycology. Blackwell Science, Oxford, pp 83-93

Huesemann M, Benemann JR (2009) Biofuels from microalgae: review of products, process and potential, with special focus on Dunaliella sp. In: Ben-Amotz A, Polle JEW, Subba-Rao VD (eds) The alga Dunaliella: Biodiversity, Physiology, Genomics and Biotechnology. Science Publishers, New Hampshire

Kumar A, Ergas S, Yuan X, Sahu A, Zhang Q, Dewulf J, Malcata FX, van Langenhove H (2010) Enhanced CO2 fixation and biofuel production via microalgae: Recent developments and future directions. Trends Biotechnol 28: 371-380

Lardon L, Hélias A, Sialve B, Steyer J-, Bernard O (2009) Life-cycle assessment of biodiesel production from microalgae. Environmental Science and Technology 43: $6475-6481$

Lau KY, Pleissner D, Lin CSK (2014) Recycling of food waste as nutrients in Chlorella vulgaris cultivation. Bioresour Technol 170: 144-151

Levine RB, Costanza-Robinson MS, Spatafora GA (2011) Neochloris oleoabundans grown on anaerobically digested dairy manure for concomitant nutrient removal and biodiesel feedstock production. Biomass Bioenergy 35: 40-49

Li Y, Chen Y, Chen P, Min M, Zhou W, Martinez B, Zhu J, Ruan R (2011) Characterization of a microalga Chlorella sp. well adapted to highly concentrated municipal wastewater for nutrient removal and biodiesel production. Bioresour Technol 102: 5138-5144

Mata TM, Martins AA, Caetano NS (2010) Microalgae for biodiesel production and other applications: A review. Renewable and Sustainable Energy Reviews 14: 217-232 
Min M, Hu B, Zhou W, Li Y, Chen P, Ruan R (2012) Mutual influence of light and $\mathrm{CO} 2$ on carbon sequestration via cultivating mixotrophic alga Auxenochlorella protothecoides UMN280 in an organic carbon-rich wastewater. J Appl Phycol 24: 1099-1105

Muñoz R, Guieysse B (2006) Algal-bacterial processes for the treatment of hazardous contaminants: A review. Water Res 40: 2799-2815

Mutanda T, Karthikeyan S, Bux F (2011) The utilization of post-chlorinated municipal domestic wastewater for biomass and lipid production by Chlorella spp. under batch conditions. Appl Biochem Biotechnol 164: 1126-1138

Olguín EJ (2012) Dual purpose microalgae-bacteria-based systems that treat wastewater and produce biodiesel and chemical products within a Biorefinery. Biotechnol Adv 30: 1031-1046

Olguín EJ (2003) Phycoremediation: Key issues for cost-effective nutrient removal processes. Biotechnol Adv 22: 81-91

Olguín EJ, Galicia S, Mercado G, Pérez T (2003) Annual productivity of Spirulina (Arthrospira) and nutrient removal in a pig wastewater recycling process under tropical conditions. J Appl Phycol 15: 249-257

Olguín EJ, Sánchez-Galván G (2010) Aquatic phytoremediation: Novel insights in tropical and subtropical regions. Pure and Applied Chemistry 82: 27-38

Órpez R, Martínez ME, Hodaifa G, El Yousfi F, Jbari N, Sánchez S (2009) Growth of the microalga Botryococcus braunii in secondarily treated sewage. Desalination 246: $625-630$

Park JBK, Craggs RJ, Shilton AN (2011) Wastewater treatment high rate algal ponds for biofuel production. Bioresour Technol 102: 35-42

Park KY, Lim B-, Lee K (2009) Growth of microalgae in diluted process water of the animal wastewater treatment plant. Water Science and Technology 59: 2111-2116

Pittman JK, Dean AP, Osundeko O (2011) The potential of sustainable algal biofuel production using wastewater resources. Bioresour Technol 102: 17-25

Rodolfi L, Zittelli GC, Bassi N, Padovani G, Biondi N, Bonini G, Tredici MR (2009) Microalgae for oil: Strain selection, induction of lipid synthesis and outdoor mass cultivation in a low-cost photobioreactor. Biotechnol Bioeng 102: 100-112

Rosenberg JN, Oyler GA, Wilkinson L, Betenbaugh MJ (2008) A green light for engineered algae: redirecting metabolism to fuel a biotechnology revolution. Curr Opin Biotechnol 19: 430-436 
Ruiz-Marin A, Mendoza-Espinosa LG, Stephenson T (2010) Growth and nutrient removal in free and immobilized green algae in batch and semi-continuous cultures treating real wastewater. Bioresour Technol 101: 58-64

Schenk PM, Thomas-hall SR, Stephens E, Marx UC, Mussgnug JH, Posten C, Kruse O, Hankamer B (2008) Second generation biofuels: high-efficiency microalgae for biodiesel production. Bioenergy Res 1: 20-43

Singh J, Gu S (2010) Commercialization potential of microalgae for biofuels production. Renewable and Sustainable Energy Reviews 14: 2596-2610

Spolaore P, Joannis-Cassan C, Duran E, Isambert A (2006) Commercial applications of microalgae. Journal of Bioscience and Bioengineering 101: 87-96

Sutherland DL, Turnbull MH, Broady PA, Craggs RJ (2014) Effects of two different nutrient loads on microalgal production, nutrient removal and photosynthetic efficiency in pilot-scale wastewater high rate algal ponds. Water Res 66: 53-62

Sydney EB, da Silva TE, Tokarski A, Novak AC, de Carvalho JC, Woiciecohwski AL, Larroche C, Soccol CR (2011) Screening of microalgae with potential for biodiesel production and nutrient removal from treated domestic sewage. Appl Energy 88: 3291-3294

Takagi M, Watanabe K, Yamaberi K, Yoshida T (2000) Limited feeding of potassium nitrate for intracellular lipid and triglyceride accumulation of Nannochloris sp. UTEX LB1999. Appl Microbiol Biotechnol 54: 112-117

Wang L, Li Y, Chen P, Min M, Chen Y, Zhu J, Ruan RR (2010) Anaerobic digested dairy manure as a nutrient supplement for cultivation of oil-rich green microalgae Chlorella sp. Bioresour Technol 101: 2623-2628

Yang J, Li X, Hu H, Zhang X, Yu Y, Chen Y (2011) Growth and lipid accumulation properties of a freshwater microalga, Chlorella ellipsoidea YJ1, in domestic secondary effluents. Appl Energy 88: 3295-3299

\section{Figure captions}

Figure 1.- Variation in biomass concentration, biomass productivity and florescence of chlorophylls in the selected strains as a function of the culture medium used: Arnon or secondary-treated wastewater (STWW).

Figure 2.- Variation in cell density and cell weight of the selected strains as a function of the culture medium used: Arnon or secondary-treated wastewater (STWW). 
Figure 3.- Variation in the biomass extinction coefficient and the average irradiance inside the cultures as a function of the culture medium used: Arnon or secondary-treated wastewater (STWW).

Figure 4.- Variation in the electron transfer rate (ETR) with the light availability for each of the strains tested as a function of the culture medium used: Arnon or secondarytreated wastewater (STWW).

Figure 5.- Variation in the biochemical composition of the biomass as a function of the culture medium used: Arnon or secondary-treated wastewater (STWW). A) Lipid content; B) Protein content; C) Carbohydrate content; D) Ash content.

Figure 6.- Variation in the fatty acid content of the biomass and the fatty acid productivity of the cultures as a function of the culture medium used (Arnon or secondary-treated wastewater-STWW) and the strain used.

Figure 7.- Variation in the nutrient content at the inlet and outlet of the culture medium reactors as a function of the culture medium used (Arnon or secondary-treated wastewater-STWW) and the strain used.

Figure 8.- Variation in the quantum yield and photosynthetic efficiency as a function of the culture medium (Arnon or secondary-treated wastewater-STWW) and the strain used.

Figure 9.- Variation in the nitrogen and phosphorus coefficient yield as a function of the culture medium (Arnon or secondary-treated wastewater-STWW) and the strain used.

Figure 10.- Variation in the normalized efficiency of the selected parameters (cell weight, biomass productivity, quantum yield, nitrogen and phosphorus coefficient yield, and fatty acid content) as a function of the culture medium (Arnon or secondary-treated wastewater-STWW) and the strain used. 